DOI: 10.18413/2518-1092-2019-4-2-0-6

Александров Д.Д.

Косов Е.О.

Архипов С.H.

МОДЕЛЬ ПРОГРАММНОГО КОМПЛЕКСА ПОДДЕРЖКИ ПРИНЯТИЯ РЕШЕНИЙ ПО ПРОЕКТИРОВАНИЮ ПАССИВНОЙ ОПТИЧЕСКОЙ СЕТИ

Федеральное государственное казённое военное образовательное учреждение высшего образования «Академия Федеральной службы охраны Российской Федерации», ул. Приборостроительная, д. 35, г. Орёл, 302034, Россия

e-mail: danillaleksandrov57@mail.ru

\begin{abstract}
Аннотация
В статье рассматривается модель программного комплекса по проектированию пассивных оптических сетей, а также требования к ней.

Ключевые слова: пассивные оптические сети; модель; программный комплекс.
\end{abstract}

\title{
UDC 621.391.63
}

Aleksandrov D.D. Cosov. E.O. Arkhipov S.N.

MODEL OF THE PROGRAM COMPLEX SUPPORT OF DECISION MAKING ON THE DESIGN OF PASSIVE OPTICAL NETWORK

Federal state military educational institution of higher professional education "Academy of the Federal security service of the Russian Federation", 35 Priborostroitelnaya St, Orel, 302034, Russia

e-mail: danillaleksandrov57@mail.ru

\begin{abstract}
Annotation
The article discusses the model of the software package for the design of passive optical networks, as well as the requirements for it.

Keywords: passive optical networks; model; software package.
\end{abstract}

\section{ВВЕДЕНИЕ}

Эффективность построения и применения пассивных оптических сетей связи достигается комплексным решением множества задач, позволяющих максимально использовать потенциальные возможности системы специальной связи в мирное и военное время. Для обеспечения успешного выполнения системой специальной связи задач, необходимо максимально эффективно использовать ресурс, предоставляемый в интересах органов государственной власти, который представляет собой совокупность взаимоувязанных элементов и среды распространения информационных сигналов [1].

Для решения задач проектирования сетей связи специального назначения на основе технологии PON необходимо обеспечить оптимальное размещение и выбор элементов, обеспечивающих процесс приема и передачи информации с заданным качеством. Благодаря разработке программного комплекса поддержки принятия решения, возможно будет оптимально распределять элементы сети, а также выбирать такую топологию сети, при которой будет выполняться «баланс» сети в целом.

Для рационализации принятия решений при проектировании пассивных оптических сетей доступа целесообразно использование оптимизационно-имитационного подхода [2].

Оптимизационно-имитационный подход основан на совместном применении имитационных и оптимизационных моделей в процессе проектирования пассивной оптической сети [2]. При этом имитационные модели используются для оценки основных характеристик пассивных оптических сетей. В частности, важным этапом проектирования пассивной оптической 
сети является расчет оптического бюджета. Рассчитанные значения оптического бюджета пассивной оптической сети является исходной информацией для формирования критериев оптимальности и ограничений в оптимизационных моделях. Целенаправленным варьированием параметров имитационной модели можно получить оптимальные и близкие к оптимальным решения.

Рассматриваемые оптимизационные задачи обобщенно могут быть сформулированы следующими соотношениями (1):

$$
\begin{aligned}
& f_{i}(X) \rightarrow \min _{X \in D}, i=\overline{1, m} \\
& D\left\{X \mid X_{i}^{\min } \leq X_{i} \leq X_{i}^{\max }, j=\overline{1, m},\right. \\
& \left.g_{s}(X) \geq 0, h_{k}(X)=0, s=\overline{1, r} ; k=\overline{1, p}\right\}
\end{aligned}
$$

где $X=\left(x_{1} \ldots x_{n}\right)$ - вектор варьируемых параметров модели;

$f_{i}(X)$ - частные критерии оптимальности;

$D$ - допустимая область, представленная ограничениями двух видов:

прямыми ( $\left.x_{j}^{\min } \leq x_{j} \leq x_{j}^{\max }\right)$ и функциональными $\left(g_{s}(X) \geq 0, h_{k}(X) \approx 0\right)$.

При этом предполагается, что критерии и ограничения (или их часть) заданы алгоритмически в виде моделирующих процедур, позволяющих по заданным значениям $X=\left(x_{1} \ldots x_{n}\right)$ получать значения $f_{i}(X), g_{p}(X), h_{k}(X)$.

Современная сеть доступа рассматривается как сложная взаимоувязанная система, в которой в единую логистическую цепь объединяются процессы передачи данных от разных источников, а также происходит распределение этих потоков до конкретных получателей [3]. Особенностью пассивных оптических сетей является высокая трудоемкость расчета энергетических составляющих, в частности оптического бюджета сети. Это связано с многообразием связей между структурными элементами систем, неоднозначностью алгоритмов расчета при различных условиях, а также большим числом учитываемых параметров. Необходимость комплексного учета данных факторов затрудняет разработку оптимизационных моделей в аналитической формулировке и приводит к использованию аппарата имитационного моделирования. При этом связи между входными и выходными параметрами задаются с помощью различных моделирующих алгоритмов в соответствии с закономерностями функционирования пассивных оптических сетей.

Практическая реализация оптимизационно-имитационного подхода при оптимизации пассивных оптических сетей предполагает решение следующих основных задач:

- построение комплексной модели пассивной оптической сети доступа;

- формирование алгоритмов преобразования исходных данных с целью направленного проведения модельных экспериментов;

- разработка имитационных моделей в процессе поиска оптимальных решений.

Перед началом поиска оптимальной структуры пассивной оптической сети осуществляются построение оптимизационной модели (определение размерности задачи, формирование критериев и ограничений), установка исходных данных для элементов сети, а также ввод параметров, использующихся для решения данной задачи. Оптимизационная модель может быть сформирована пользователем в диалоговом режиме или выбрана из ранее разработанных моделей. Характеристики построенной модели являются исходной информацией для выбора рациональных процедур оптимизации. Далее после расчета оптического бюджета сети, пользователь может наглядно определить направления сети, где необходима корректировка параметров или изменение варианта размещения и соединения элементов. 
Применение программного комплекса поддержки принятия решений при оптимизации пассивных оптических сетей, разработанного с использованием оптимизационно-имитационного подхода, позволит учитывать различные аспекты, возникающие при выборе оптимальной с точки зрения топологической, энергетической и экономической составляющих структуры сети [4].

С целью определения содержания программного комплекса необходимо сформировать требования к массиву выходных данных, интерпретация которых позволит четко решать поставленную перед программой задачу, при этом вывод результатов должен обладать наглядностью и однозначностью. Для этого необходимо разработать модель задачи, которая будет наглядно отображать содержательную часть программного комплекса, а также определять взаимосвязь входящих в него блоков.

Для разработки модели программного комплекса, с помощью которого будет возможно рассчитать сбалансированность $P O N$ сети, необходимо создать совокупность взаимоувязанных массивов исходных данных для сети, блоков моделирования и расчета сети, а также разработать удобный и наглядный визуальный интерфейс.

Согласно поставленной задаче, модель должна состоять из взаимоувязанных блоков, представленных на рисунке 1 .

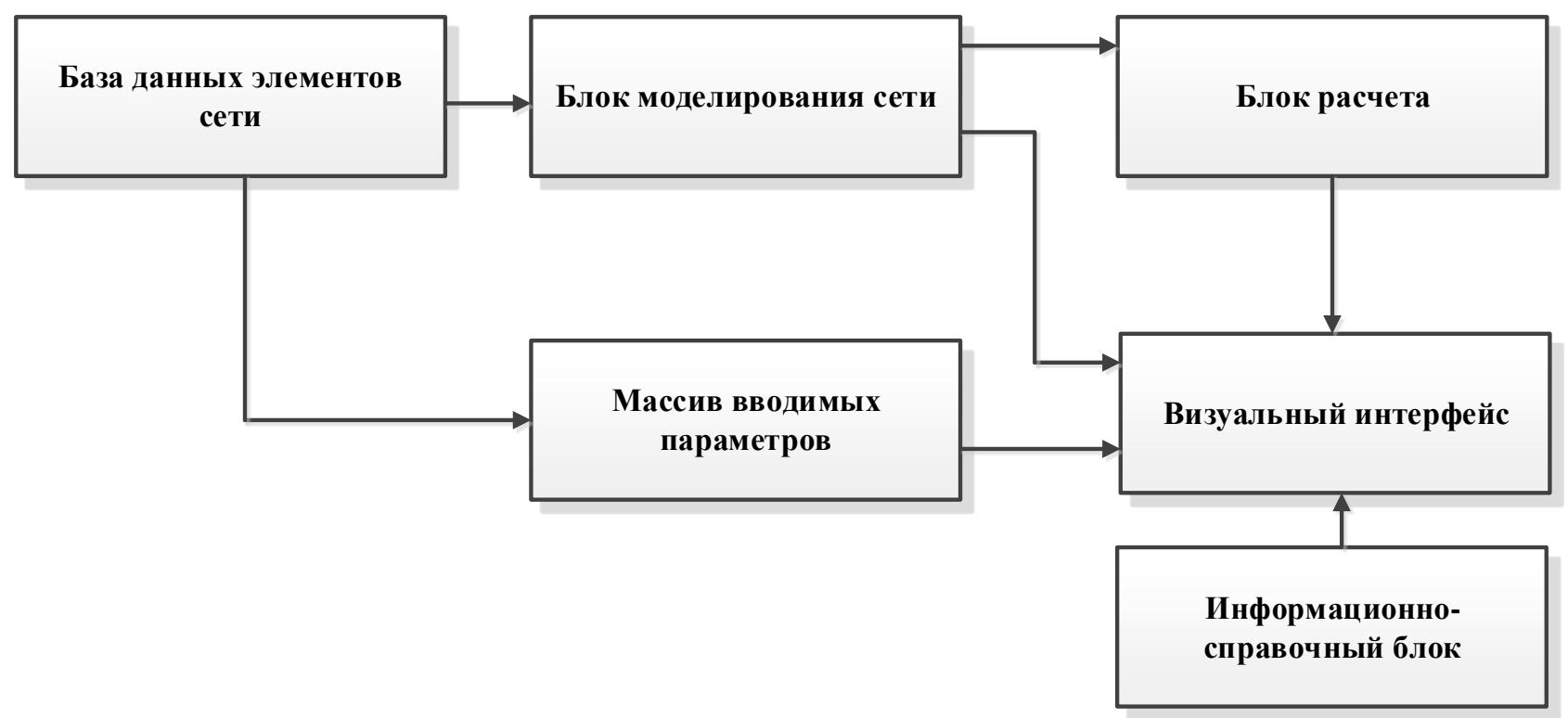

Puc. 1. Модель программного комплекса

Fig. 1. Model of the software package

В массиве вводимых параметров должны находиться все необходимые для расчета $P O N$ сети данные.

В базе данных элементов сети должно быть реализовано хранение и добавление характеристик элементов $P O N$ сети, необходимых для расчета.

Блок расчета предназначен для того, чтобы на основе вводимых параметров и модели реализовать расчет энергетического бюджета $P O N$ сети.

Для удобства ввода исходных данных и наглядности получения результатов вычисления, блок расчета должен основываться на структурированной модели сети. В блоке моделирования сети должна быть реализована возможность размещения элементов сети на рабочей области с соблюдением их топологической взаимосвязи. Также для каждого добавляемого элемента сети должны быть определены параметры, необходимые для расчета. 
С помощью визуального интерфейса программного комплекса осуществляется ввод исходных данных и моделирование сети для дальнейшего расчета энергетического бюджета и вывода результатов.

Информационно-справочный блок предназначен для того, чтобы специалист мог оперативно ознакомиться с основами технологии $P O N$ и порядком осуществления расчетов.

Основной задачей разработки программного комплекса является расчет энергетического бюджета сети доступа и ее анализ по критерию «баланса».

С целью решения поставленной задачи, необходимо чтобы выходной массив данных содержал сведения по энергетическим характеристикам каждого направления и имел возможность проводить обобщение данных с одновременным анализом указанного критерия.

Программные комплексы поддержки принятия решений (ПКППР) служат для помощи должностным лицам (ДЛ) в реализации процессов поддержки решения посредством автоматизации как творческих процессов выработки и принятия решений, так и нетворческих процессов сбора и доведения информации, образовавшиеся в результате слияния информационных систем управления и систем управления базами данных [5]. Они являются интерактивными автоматизированными системами, одной из основных задач которых помогать специалисту путем рекомендации в качестве решения наиболее рациональные альтернативы. Такие комплексы обладают простым интерфейсом, что бы у большинства пользователей не было проблем с использованием системы.

ПКППР должны быть максимально приспособлены для решения сложных, многокритериальных задач в различных сферах и призваны оказывать помощь специалисту в оптимальном выборе какого-либо ответственного решения. Такое решение основывается на анализе специалистом альтернатив, выработанных ПКППР.

В теории принятия решений [5] альтернативами называются варианты выбора возможных решений. Использование понятия «поддержка» (принятия решений) означает, что окончательный выбор решения определяет специалист, а программный комплекс лишь помогает принять наиболее оптимальное решение, давая рекомендации.

Для принятия оптимального решения по построению сети, необходимо чтобы ДЛ с использованием ПКППР мог осуществить следующие действия: задачи);

- определить множество альтернатив (возможных вариантов решения поставленной

- определить множество критериев, по которым будут оцениваться альтернативы;

- выработать оценки альтернатив по критериям;

- выбрать альтернативу, которая определяется программным комплексом поддержки принятия решений в качестве оптимального варианта решения поставленной задачи.

Определив требования к разрабатываемому программному комплексу необходимо осуществить анализ и выбор средств его реализации.

Таким образом, необходимо разработать программный комплекс, с помощью которого будет возможно моделирование сети, а также, на основе модели сети, произвести расчет энергетического бюджета с возможностью наглядным образом оценить сбалансированность сети по критерию «баланса». Также, на основе полученных данных, проектировщик должен иметь возможность оперативного изменения параметров, элементов и топологии сети для достижения необходимого оптимального значения.

\section{Список литературы}

1. Бутусов М.М., Волоконно-оптические системы передачи: Учебник для вузов. - Москва: Радио и связь, 1992. -416 с.

2. Хоботов Е.Н., Оптимизационно-имитационный подход к моделированию сложных систем Москва: Изв. РАН, 1996. - 178 с. 
3. Никульский И.Е., Оптические интерфейсы цифровых коммутационных станций и сети доступа. Москва: Техносфера, 2006. -256 с.

4. Архипов С. Н., Алгоритм оптимизации бюджета PON сети / С. Н. Архипов, И. С. Полянский // Телекоммуникации. - Специальный выпуск. Москва, 2012. - с. 3 - 9.

5. Есиков О.В., Автоматизированные информационные системы: методы построения и исследования. Модели и методы поддержки принятия решений - Москва: Инфра-М, 2010. - 488 с.

\section{References}

1. Butusov MM, Fiber-optical transmission systems: A textbook for universities. - Moscow: Radio and communications, $1992 .-416 \mathrm{c}$.

2. Khobotov, EN, Optimization and Simulation Approach to Modeling Complex Systems - Moscow: Izv. RAS, 1996. - $178 \mathrm{p}$.

3. Nikulsky I.E., Optical interfaces of digital switching stations and access networks. - Moscow: Technosphere, 2006. $-256 \mathrm{p}$.

4. Arkhipov S.N., Algorithm of budget optimization of the PON network / S.N. Arkhipov, I.S. Polyansky // Telecommunications. - Special issue. Moscow, 2012. - p. 3-9.

5. Esikov OV, Automated information systems: methods of construction and research. Decision Support Models and Methods - Moscow: Infra-M, 2010. - 488 p.

Александров Даниил Дмитриевич, студент Академии Федеральной службы охраны Российской Федерации Косов Егор Олегович, студент Академии Федеральной службы охраны Российской Федерации Архипов Сергей Николаевич, кандидат технических наук, сотрудник Академии Федеральной службы охраны Российской Федерации

Aleksandrov Daniil Dmitrievich, student Academy of the Federal security service of the Russian Federation Kosov Egor Olegovich, student Academy of the Federal security service of the Russian Federation Arkhipov Sergey Nikolaevich, candidate of technical sciences, Academy of the Federal security service of the Russian Federation 